\title{
APPLICATION OF TECHNOLOGY SURVEILLANCE TOOLS IN THE SURVEY OF OPEN SOURCE TECHNOLOGIES THAT ARE APPLICABLE IN THE TEACHING OF ENGINEERING
}

\author{
J.S. Pavlicevic ${ }^{1}$, M. Guagliano' ${ }^{1}$ J.E. Tornillo ${ }^{1}$, D. Servetto ${ }^{1}$ \\ ${ }^{1}$ School of Engineering, National University of Lomas de Zamora (ARGENTINA)
}

\begin{abstract}
Nowadays, Information and Communication Technologies (ICT) play a major role in organizations. This is particularly so for universities, that should incorporate ICT to their teaching and management processes, in order to enhance their quality of education. The implementation of open source software should be a priority for universities for a number of features, namely low cost and the possibility to run, copy, modify and distribute the software without limitations, which contributes to collaborative knowledge construction. With regards to technology surveillance, it is a discipline that allows the monitoring and identification of key technologies that, on term, permit the identification of its state of the arts at the global level.

This work is an exploratory research on the several forms of open code software that can be applied to the teaching of engineering through the use of technology surveillance tools. The findings hope to contribute to the teaching and learning processes of several training paths for the training courses of the School of Engineering of the Universidad Nacional de Lomas de Zamora (FI-UNLZ).
\end{abstract}

Keywords: Open Source Software, Education, Engineering, Technology Surveillance

\section{INTRODUCTION}

En el contexto actual en el cual nos encontramos convivimos frecuentemente con mucha información alrededor, que genera un efecto de infoxicación en el ser humano [1].

En particular, en el marco de los procesos de enseñanza y aprendizaje resulta esencial la implementación de las Tecnologías de la Información y la Comunicación (TIC) para garantizar la calidad Educativa en las Universidades [2]. Es necesario inculcar en los estudiantes, en particular de carreras de ingeniería, la importancia del uso de las nuevas tecnologías para la construcción del conocimiento y asegurar el aprendizaje de las habilidades necesarias para explorar y explotar nuevas oportunidades y de esa manera contribuir con el desarrollo social, económico, tecnológico y ambiental (A Ali, L Aliyar 2012).

El software es cada vez más el gran intermediario entre la información y la inteligencia humana (Hernández, J. M. 2005). Si bien existen claras discrepancias a nivel conceptual entre los softwares de código abierto (Open Source) y los softwares propietarios, también es importante diferenciar los conceptos "Open Source" de "Free Software", que pueden ser interpretados como sin costo o bien como libre. En esencia, el software open source presenta ventajas significativas cuando se trabaja en entornos colaborativos como son las Universidades, ya que se generan a partir de la cooperación y los aportes de los propios usuarios. Además, al no tener costo de adquisición, la implementación de este tipo de software resulta atractiva para las organizaciones en general y las Universidades en particular, ya que de esta manera pueden optimizar el uso de su presupuesto que es, en muchos casos, dinero público.

Según definió Stallman en el año 2005 (STALLMAN, (2005) el concepto de software libre es un asunto de libertad y no de precio. Además, para que un software sea considerado como libre debe garantizar a la comunidad 4 libertades fundamentales:

0. La libertad cero es aquella relacionada con ejecutar el software con cualquier propósito y de la manera que se desee.

1. La libertad número uno refiere a la posibilidad del usuario de modificarlo para ajustarlo a sus necesidades.

2. La libertad número dos es la de ayudar a la comunidad mediante la libre distribución. 
3. La tercera y última libertad es la de aportar a la comunidad mediante la publicación de versiones mejoradas de modo que todos puedan beneficiarse de su trabajo.

Además, en el caso particular de las Universidades, existe una responsabilidad aun mayor al momento de implementar softwares, para cualquier propósito. Esto se debe a que las instituciones educativas deben garantizar su uso responsable y para fines educativos. Además, dichas Unidades Académicas deben exigir que el software opere en la lengua propia de donde se vaya a implementar (localización), garantice el acceso a la información en todo momento (perenidad) y no permita que personas no autorizadas tengan acceso a datos confidenciales o información reservada (seguridad) (GARCÍA A Y CUELLO R 2007).

Es por ello que las Universidades deben estar constantemente alerta sobre los nuevos desarrollos que se realizan en el campo del software libre. En este sentido, resulta fundamental mejorar los procesos de búsqueda, recolección, depuración y análisis de la información para garantizar que sea realmente pertinente, de calidad y se obtenga de manera eficiente. Para ello se deben emplear diversas herramientas como es el caso de la Vigilancia Tecnológica y la Inteligencia Estratégica (VTelE); disciplina que tiene lugar a raíz de la creciente utilización de las Tecnologías de la Información y la Comunicación (TIC) (ESCORSA, P. MASPONS, R. 2001).

La Vigilancia Tecnológica se define según la norma nacional IRAM 50520-2017 como un proceso sistemático y organizado que tiene un rol fundamental en la gestión de la investigación, desarrollo e innovación de las organizaciones. Además, se considera una herramienta indispensable para la búsqueda y recolección de información y para identificar cambios, novedades y oportunidades de un mercado o sector. Por su parte el concepto de Inteligencia Estratégica implica estar al servicio de las decisiones estratégicas, y abarca tanto la inteligencia económica como la gestión del conocimiento (MINCYT 2015). A su vez, es una instancia que tiene lugar a continuación de la Vigilancia Tecnológica y comprende el análisis, la interpretación y la comunicación del conocimiento generado a partir de los datos. Este conocimiento suele comprender aspectos científicos, tecnológicos, normativos y de mercado; y es gestionado por los responsables de la toma de decisiones estratégicas de las organizaciones (IRAM 50520-2017).

Por lo tanto, la utilización de estas herramientas permite fortalecer los procesos de innovación y de toma de decisiones estratégicas de las organizaciones y gestionar los cambios del entorno de manera anticipada (GUAGLIANO, M. 2014).

Este trabajo es una continuación de la recopilación de softwares de código abierto realizada en el año 2017 desde la Unidad de Vigilancia e Inteligencia Estratégica (VINES) de la Facultad de Ingeniería de la Universidad Nacional de Lomas de Zamora (Argentina) (TEYET 2017) orientada a profundizar en aquellos softwares relacionados con la gestión de proyectos, la investigación de operaciones, y la planificación de la producción y el abastecimiento. Para ello se emplean metodologías, técnicas y herramientas propias de las actividades de Vigilancia Tecnológica e Inteligencia Estratégica; las cuales han permitido elaborar una matriz que refleja las potencialidades y limitaciones de cada software y sirve como insumo para decidir sobre su aplicación -o no- en las ramas del conocimiento mencionadas de las carreras de Ingeniería que se dictan en la Unidad Académica.

\section{METHODOLOGY}

La metodología que se utiliza desde la unidad VINES es la desarrollada por el Ministerio de Ciencia, Tecnología e Innovación Productiva (MINCYT) en el año 2015 (ALTEC 2017), que es una adaptación de la norma UNE 166006:2011. La misma consta de 5 instancias fundamentales de trabajo: Planificación, Búsqueda y Recolección, Análisis y Validación, Difusión y Protección, Toma de Decisión. Luego, se deben evaluar los resultados y volver a iterar el proceso. Por lo mencionado anteriormente, y teniendo en cuenta que esta disciplina es un proceso sistemático, se la suele representar como un ciclo. En consecuencia, el ciclo de la Vigilancia Tecnológica y la Inteligencia Estratégica se muestra en la Fig. 1. 


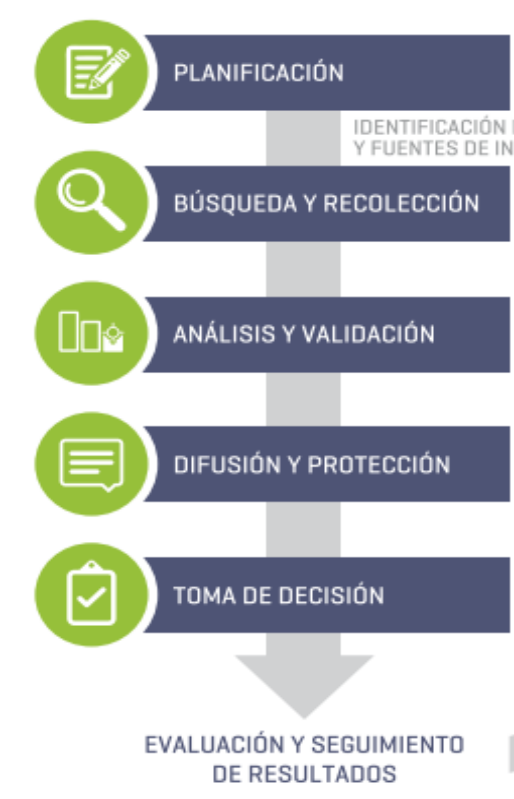

Figure 1. Metodología utilizada: Ciclo de la Vigilancia y la Inteligencia. Fuente: MINCyT 2015.

Las etapas de planificación y búsqueda y recolección de información se han llevado a cabo en la primera etapa durante el año 2017 (TEYET 2017), la cual tuvo un objetivo de búsqueda global orientado a la enseñanza de la Ingeniería en general. Como el objetivo de este trabajo es más específico, se decide comenzar profundizando en estas mismas etapas modificando el objetivo de búsqueda. Para ello se relevan las necesidades específicas de los departamentos involucrados y luego se realizan las ecuaciones de búsqueda, a partir de palabras clave identificadas. A continuación, se aplican en diversos softwares y bases de datos. Esencialmente se utilizan metabuscadores, multibuscadores y bases de datos de patentes y publicaciones científicas. Las herramientas informáticas utilizadas se muestran en la Fig. 2.

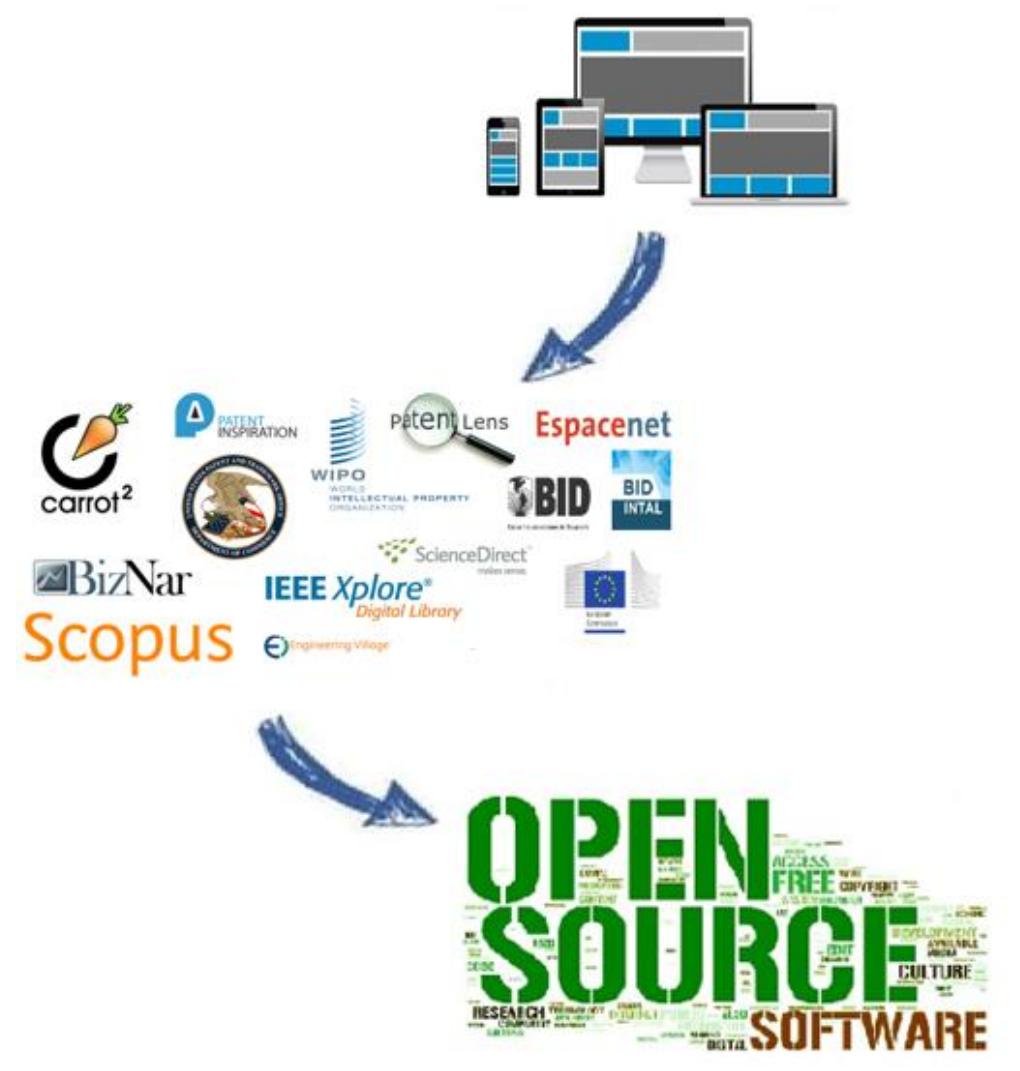

Figure 2. Herramientas utilizadas para la búsqueda y recolección de información. Fuente: elaboración propia. 
Luego de haber recolectado más de 50 softwares open source específicos para estas ramas del conocimiento seleccionadas para el presente trabajo, se han investigado sus potencialidades, limitaciones y principales aplicaciones. Finalmente se ha elaborado una matriz de comparación para luego realizar la validación con los jefes de departamento y cátedras. De esta manera, se proporciona toda la información necesaria para que luego puedan tomar las decisiones más acertadas respecto a qué software implementar en cada asignatura.

\section{RESULTS}

Del corpus total de resultados obtenidos, se han seleccionado más de 30 software open source que, debido a factores como su alcance, disponibilidad y usabilidad han resultado aptos para su aplicación en la currícula de Ingeniería, según el criterio de la institución. Dichos resultados se dividen en dos grupos principales: El listado de softwares seleccionados con sus potencialidades y por otro lado las instituciones líderes en aplicación de dichos softwares, indicando además la manera en que lo hacen. Por cuestiones de extensión del documento, se mostrarán solo los resultados asociados a alguna de las categorías a modo de ejemplo. Vale destacar que se ha realizado el mismo trabajo para todas las categorías mencionadas al inicio del documento.

\subsection{Softwares seleccionados y potencialidades}

En la tabla 1 se muestra la distribución de los software relevados según su campo de aplicación.

Table 1. Cantidad de software relevados según su campo de aplicación

\begin{tabular}{l|c}
\hline \hline & Software \\
\hline Project Management & 11 \\
\hline Operational Research & 14 \\
\hline Supply and Production Planning & 12 \\
\hline
\end{tabular}

Luego, para cada área del conocimiento se han detallado ciertos atributos específicos deseables en el software. A modo de ejemplo, se muestra en la Figura 4 una tabla comparativa entre algunos de los software relevados que resuelven problemáticas de la gestión de proyectos.

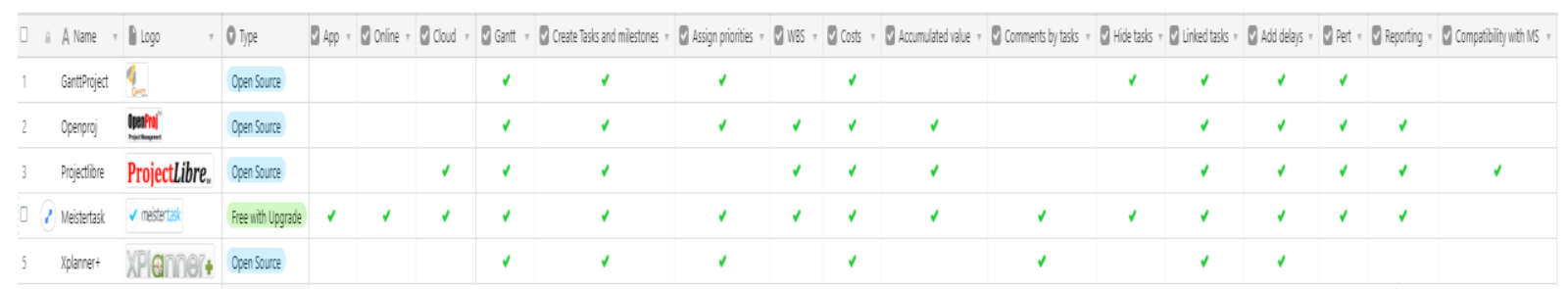

Figure 4: Listado de software seleccionados y potencialidades.

De los 5 softwares mencionados en la Fig. 4 solo hay uno que no es open source; sino que se ofrece una versión gratuita con funcionalidades limitadas y la posibilidad de elevar las capacidades del software realizando un upgrade por el cual hay que pagar. Es importante destacar que ninguna de las alternativas open source tiene la posibilidad de trabajar online, lo cual es por un lado una limitación, y por otro lado una oportunidad de desarrollo. Con respecto a las funcionalidades específicas relacionadas con el campo de aplicación, se concluye que todos cumplen con los requisitos básicos para realizar diagramas de Gantt y planificación de tareas. No obstante, resulta importante ponderar cuestiones como la elaboración de Work breakdown structure (WBS), la elaboración de reportings y la compatibilidad con otras plataformas para poder tomar la decisión más acertada.

\subsection{Instituciones líderes y principales aplicaciones}

Por otra parte, se han identificado instituciones cuyo background en el uso de estos softwares es muy elevado. Además, se muestran sus principales publicaciones científicas relacionadas tanto con las 
experiencias de aplicación como también con futuros desarrollos y mejoras previstas para los distintos tipos de software. Algunas de las publicaciones seleccionadas se muestran en la Fig. 5.

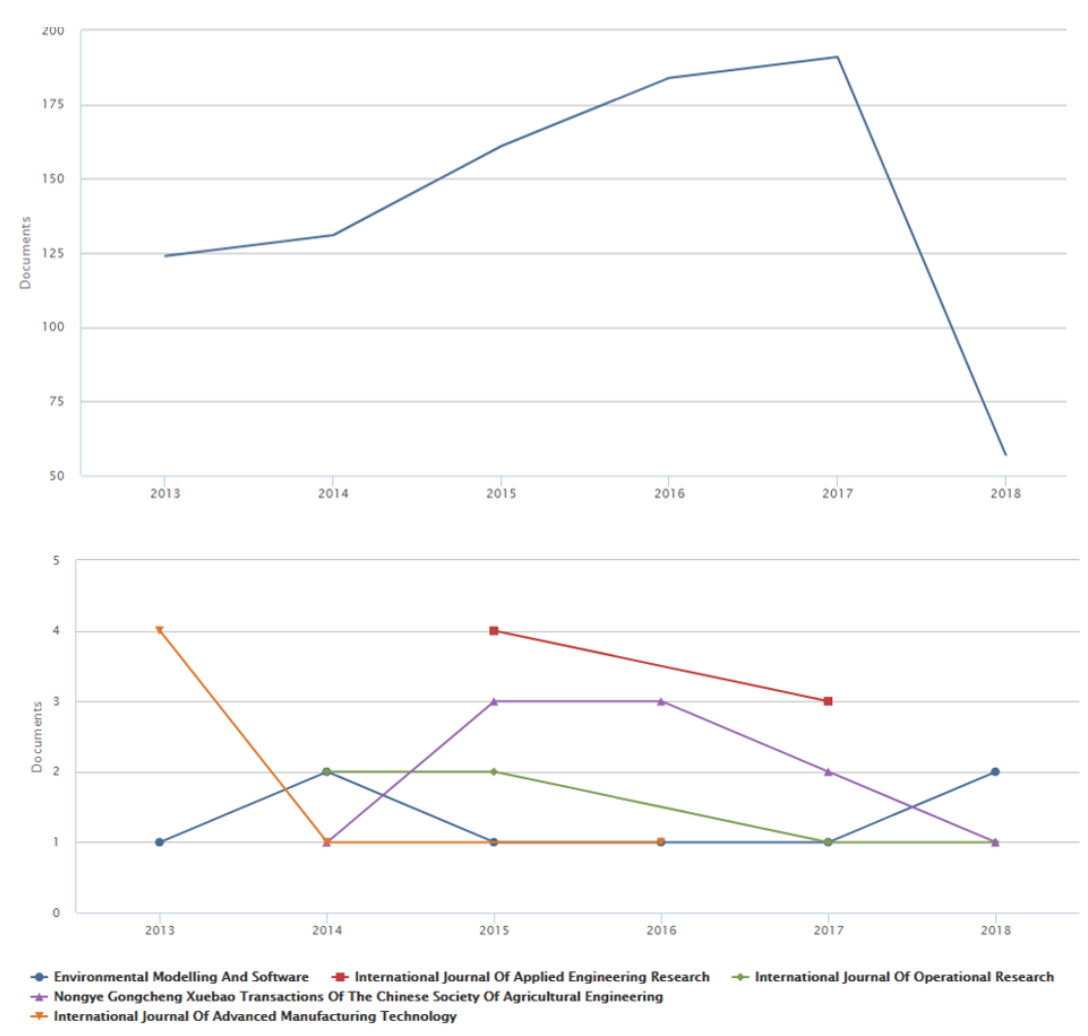

Figure 5: Cantidad de publicaciones y revistas más relevantes

Se ha encontrado un corpus total de 848 documentos relacionados con los software open source mencionados aplicados en investigación operativa; que además están vinculados con la educación. Como se puede observar, la evolución en cantidad de publicaciones es creciente y las revistas tienen como principales actores a universidades de China y Estados Unidos. Cabe destacar que estos datos fueron extraídos a principios del año 2018. Además, se ha proporcionado un listado de publicaciones destacadas y de autores principales con el fin de poder realizar un seguimiento eficiente del avance del estado del arte de la temática.

\section{CONCLUSIONS}

Partiendo del relevamiento de Software Open Source aplicables en la enseñanza de la ingeniería realizado por la unidad VINES en el año 2017, en trabajo se ha profundizado en aquellas tecnologías que resuelven problemáticas de un conjunto de ramas del conocimiento específicas. El objetivo principal propuesto fue brindarle a la institución en general y a los responsables de departamento en particular información pertinente y de calidad relacionada con las distintas tecnologías existentes que se aplican a nivel global y regional en la enseñanza de carreras de perfil tecnológico, como es el caso de las ingenierías. Las mismas han permitido mejorar los niveles de calidad de enseñanza. En este sentido, se han relevado y estructurado más de 30 softwares, detallando sus potencialidades, limitaciones y además se han identificado las instituciones líderes en implementación y desarrollo de los mismos. Por todo lo expuesto se concluye que el objetivo ha sido alcanzado satisfactoriamente.

Por otra parte, existe un valor adicional en este trabajo. El mismo radica en que se considera que el desarrollo, la promoción y la difusión de las tecnologías de código abierto por parte de las instituciones educativas motivan la generación innovaciones tecnológicas por parte de los estudiantes, fomentan el trabajo colaborativo entre los investigadores y también contribuyen a la consolidación de una sociedad del conocimiento más libre.

Como trabajos futuros se propone replicar este trabajo en otras ramas del conocimiento, en línea con las necesidades que sean planteadas desde los distintos órganos de la institución. Esto permitiría diseñar nuevas soluciones didácticas que eleven los procesos de enseñanzas educativas en carreras de ingeniería. Por último, se destaca el valor agregado del presente trabajo, como experiencia que se 
puede ser replicada desde lo metodológico hasta las soluciones y resultados obtenidos, por otras instituciones que tengan carreras de ingeniería.

\section{REFERENCES}

[1] 